\title{
Multiple Solutions for a Class of Semilinear Elliptic Equations with Nonlinear Boundary Conditions
}

\author{
Ziyan Yao \\ Department of Mathematics, University of Shanghai for Science and Technology, Shanghai, China \\ Email: ziyanyao160@163.com
}

Received October 23, 2013; revised November 23, 2013; accepted November 30, 2013

Copyright (c) 2014 Ziyan Yao. This is an open access article distributed under the Creative Commons Attribution License, which permits unrestricted use, distribution, and reproduction in any medium, provided the original work is properly cited. In accordance of the Creative Commons Attribution License all Copyrights (C) 2014 are reserved for SCIRP and the owner of the intellectual property Ziyan Yao. All Copyright (C) 2014 are guarded by law and by SCIRP as a guardian.

\section{ABSTRACT}

In this paper, using Local Linking Theorem, we obtain the existence of multiple solutions for a class of semilinear elliptic equations with nonlinear boundary conditions, in which the nonlinearites are compared with higher Neumann eigenvalue and the first Steklov eigenvalue.

\section{KEYWORDS}

\section{Multiple Solutions; Nonlinear Boundary Conditions; Local Linking Theorem}

\section{Introduction}

In this paper, we investigate the multiple solutions for semilinear elliptic equation with nonlinear boundary conditions

$$
\begin{cases}-\Delta u+c(x) u=f(x, u), & x \in \Omega \\ \frac{\partial u}{\partial v}=g(x, u), & x \in \partial \Omega\end{cases}
$$

where $\Omega \subset \mathbb{R}^{N}(N \geq 3)$ is bounded domain with smooth boundary $\partial \Omega$ and $\frac{\partial}{\partial v}=v \cdot \nabla$ is the outward normal derivative on $\partial \Omega$, and the function $c(x)$ satisfies

$$
\text { C) } c(x) \in L^{\infty}(\Omega) \text {, and } c(x) \geq 0 \text { a.e. on } \Omega \text { with } \int_{\Omega} c(x) \mathrm{d} x>0 \text {. }
$$

Problems of the above type have been discussed extensively. In 1902, Steklov (see [1]) studied the eigenvalue problem

$$
\left\{\begin{array}{l}
-\Delta u=0, x \in \Omega \\
\frac{\partial u}{\partial v}=\lambda u, x \in \partial \Omega
\end{array}\right.
$$

Auchmuty (see [2]) considered the eigenvalue problem

$$
\begin{cases}-\Delta u(x)+c(x) u=0, & x \in \Omega \\ \frac{\partial u}{\partial v}=\lambda u(x), & x \in \partial \Omega\end{cases}
$$

where $c(x)$ satisfies the condition $C$ ) and proved that the eigenfunctions provide a complete orthonormal 
bases of certain closed subspace of $H^{1}(\Omega)$. Using sub and super-solutions method, Amann (see [3]), Mawhin and Schmitt (see [4]) obtained some existence results for the problem (1.1). However, since it is based on comparison techniques, the sub and super-method does not apply when the nonlinearities are compared with higher eigenvalues.

In this paper, using the Local Linking Theorem, we obtain multiple solutions for the problem (1.1), which the nonlinearites are compared with higher Neumann eigenvalue and the first Steklov eigenvalue.

\section{Preliminaries and Main Results}

Let $L^{p}(\Omega)$ denote the Lebesgue space with the norm $|u|_{p}=\left(\iint_{\Omega}|u|^{p} \mathrm{~d} x\right)^{\frac{1}{p}}$, and $L^{p}(\partial \Omega, \mathrm{d} \sigma)$ with the norm $|u|_{p, \partial \Omega}=\left(\int_{\partial \Omega}|u|^{p} \mathrm{~d} \sigma\right)^{\frac{1}{p}}$. Obviously, the space $L^{2}(\Omega)$ and the space $L^{2}(\partial \Omega, \mathrm{d} \sigma)$ inner product are denoted by

$$
(u, v)=\int_{\Omega} u v \mathrm{~d} x,(u, v)_{\partial}=\int_{\partial \Omega} u v \mathrm{~d} \sigma .
$$

$H^{1}(\Omega)$ is a Hilbert space under the standard inner product

$$
[u, v]_{1}=\int_{\Omega}(u(x) v(x)+\nabla u(x) \nabla v(x)) \mathrm{d} x,
$$

with the associated norm $\|u\|_{1}$. As the function $c(x)$ satisfies the condition $\left.C\right)$, we define the weighted $H^{1}-c$ - inner product by

$$
[u, v]_{c}=\int_{\Omega}(c(x) u(x) v(x)+\nabla u(x) \nabla v(x)) \mathrm{d} x,
$$

and the associated norm $\|u\|_{c}$. By Corollary 3.3 in [2], we obtain that the norm $\|u\|_{c}$ is equivalent to the standard norm $\|u\|_{1}$. As the function $c(x)$ satisfies the condition $\left.C\right)$, by Equation (2.2), we can split

$$
H^{1}(\Omega)=H_{0}^{1}(\Omega) \oplus_{c}\left[H_{0}^{1}(\Omega)\right]^{\perp},
$$

as a direct orthogonal sum.

Now, we state the Local Linking theorem introduced by [5].

Lemma 2.1 Let $X$ is a reflexive Banach space, $X=Y \oplus V$ with $\operatorname{dim} V<+\infty, \Phi \in C^{1}(X, \mathbb{R})$ satisfies the (PS) condition, if

1) there exists a constant $\rho>0$ such that

$$
\begin{aligned}
& \Phi(x) \leq 0, \forall x \in V \text { with }\|x\|_{V} \leq \rho, \\
& \Phi(x) \geq 0, \forall x \in Y \text { with }\|x\|_{Y} \leq \rho,
\end{aligned}
$$

2) $\Phi$ is bounded below and $\inf _{X} \Phi<0$, then the functional $\Phi$ has at least two nontrivial critical points.

Proof. See Theorem 4 in [5].

For the problem (1.3), Auchmuty (see [2]) obtained that

$$
\mu_{1} \oint_{\partial \Omega}(\Gamma u)^{2} \mathrm{~d} \sigma \leq \int_{\Omega}\left(|\nabla u|^{2}+c(x) u^{2}\right) \mathrm{d} x,
$$

holds for all $u \in H^{1}(\Omega)$, where $\mu_{1}$ is the first Steklov eigenvalue for the problem (1.3). In [6], for the Neuman eigenvalue problem

$$
\begin{cases}-\Delta u(x)+c(x) u=\lambda u(x), & x \in \Omega, \\ \frac{\partial u}{\partial v}=0, & x \in \partial \Omega,\end{cases}
$$


they obtain that the above problem has a sequence of real eigenvalues

$$
0<\lambda_{1} \leq \lambda_{2} \leq \cdots \leq \lambda_{j} \leq \cdots \rightarrow \infty \text {, as } j \rightarrow \infty,
$$

with finite dimensional eigenspaces.

Assume that, $f: \Omega \times \mathbb{R} \rightarrow \mathbb{R}, g: \bar{\Omega} \times \mathbb{R} \rightarrow \mathbb{R}$ are Carathedory functions satisfying

H1) There exist $c_{1}>0$ and $2<q_{1}<2^{*}=\frac{2 N}{N-2}$ such that

$$
|f(x, t)| \leq c_{1}\left(1+|t|^{q_{1}-1}\right),
$$

for all $t \in \mathbb{R}$ and for a.e. $x \in \Omega$;

H2) There exist $c_{2}>0$ and $2<q_{2}<2_{*}=\frac{2(N-1)}{N-2}$ such that

$$
|g(x, t)| \leq c_{2}\left(1+|t|^{q_{2}-1}\right),
$$

for all $t \in \mathbb{R}$ and for a.e. $x \in \partial \Omega$;

H3) There exist $\lambda, \mu>0$ such that

$$
\liminf _{|u| \rightarrow \infty} \frac{g(x, u)}{u} \leq \limsup _{|u| \rightarrow \infty} \frac{g(x, u)}{u} \leq \mu<\mu_{1}
$$

and

$$
\liminf _{|u| \rightarrow \infty} \frac{f(x, u)}{u} \leq \limsup _{|u| \rightarrow \infty} \frac{f(x, u)}{u} \leq \lambda<\lambda_{1},
$$

uniformly for $x \in \bar{\Omega}$ with

$$
\lambda_{1} \mu+\mu_{1} \lambda<\mu_{1} \lambda_{1} .
$$

H4) There exist a integer $k \geq 1, \delta>0$ and four constants $\alpha, \beta, a, b \in \mathbb{R}$ such that

$$
\frac{1}{2} a t^{2} \leq G(x, t) \leq \frac{1}{2} b t^{2} \text { and } \frac{1}{2} \alpha t^{2} \leq F(x, t) \leq \frac{1}{2} \beta t^{2}
$$

uniformly for $x \in \bar{\Omega}, \quad 0<|t|<\delta$, where $F(x, t)=\int_{0}^{t} f(x, s) \mathrm{d} s, G(x, t)=\int_{0}^{t} g(x, s) \mathrm{d} s$,

$$
\lambda_{k}<\alpha \leq \beta<\lambda_{k+1}, \lambda_{k} \mu_{1}<\lambda_{k} a+\mu_{1} \alpha \text { and } \lambda_{k+1} b+\mu_{1} \beta<\lambda_{k+1} \mu_{1}
$$

Theorem 2.2 Suppose $c(x)$ satisfies C), and H1)-H4) hold, Then the problem (1.1) has at least two distinct nontrivial solutions.

\section{The Proofs of Theorem 2.2}

Now, we define the functional $\Phi: H^{1}(\Omega) \rightarrow \mathbb{R}$

$$
\Phi(u)=\frac{1}{2} \int_{\Omega}\left(|\nabla u|^{2}+c(x) u^{2}\right) \mathrm{d} x-\int_{\Omega} F(x, u) \mathrm{d} x-\oint_{\partial \Omega} G(x, u) \mathrm{d} \sigma=\frac{1}{2}\|u\|_{c}^{2}-J(u),
$$

where $J(u)=\int_{\Omega} F(x, u) \mathrm{d} x+\oint_{\partial \Omega} G(x, u) \mathrm{d} \sigma$.

Since the function $f(x, u)$ satisfies H1), $g(x, u)$ satisfies H2), by the Sobolev embedding of $H^{1}(\Omega)$ into $L^{\frac{2 N}{N-2}}(\Omega)$, the continuity of the trace operator from $H^{1}(\Omega)$ into $L^{\frac{2(N-1)}{N-2}}(\partial \Omega)$ and the Holder inequality, we obtain that the functional $\Phi(u)$ is well defined. Moreover, by Lemma 2.1, and Lemma 4.2 in [7], we obtain that $\Phi \in C^{1}\left(H^{1}(\Omega), \mathbb{R}\right)$, and 


$$
\Phi^{\prime}(u) v=\int_{\Omega}(\nabla u \nabla v+c(x) u v) \mathrm{d} x-\int_{\Omega} f(x, u) v \mathrm{~d} x-\oint_{\partial \Omega} g(x, u) v \mathrm{~d} \sigma, \forall v \in H^{1}(\Omega) .
$$

Furthermore, the functional $J(u)$ is weakly continuous, and $J^{\prime}(u)$ is compact. Let $u=v$ in (3.2) and a simple computation, we obtain that the critical point of the functional $\Phi(u)$ is the weak solution of the problem (1.1).

Lemma 3.1 (see [7]) Assume that the function $c(x)$ satisfies the condition C), H1) and H2) hold. If $\left\{u_{m}\right\}$ is a (PS) sequence for the functional $\Phi$, and $\left\{u_{m}\right\}$ is bounded in $H^{1}(\Omega)$, then $\left\{u_{m}\right\}$ has a strongly convergence subsequence. i.e. $\Phi(u)$ satisfies the (PS) condition.

Lemma 3.2 Assume that $c(x)$ satisfies the condition C), and H1)-H3) hold, the functional $\Phi(u)$ is coercive on $H^{1}(\Omega)$.

Proof By H3), we obtain that there exist some constants $0<\varepsilon<\lambda, 0<\varepsilon<\mu$, and $M_{1}>0, C>0$ such that

$$
F(x, t) \leq \frac{1}{2}(\lambda-\varepsilon) t^{2}+c, \forall|t| \geq M_{1}, G(x, t) \leq \frac{1}{2}(\mu-\varepsilon) t^{2}+c, \forall|t| \geq M_{1} .
$$

From H1), H2) and Equation (3.3), we obtain that there exist $f_{0} \in L^{1}(\Omega), g_{0} \in L^{1}(\partial \Omega)$ such that

$$
F(x, u) \leq \frac{1}{2}(\lambda-\varepsilon) u^{2}+f_{0}, G(x, u) \leq \frac{1}{2}(\mu-\varepsilon) u^{2}+g_{0},
$$

Hence, we obtain that

$$
\begin{aligned}
\Phi(u) & =\frac{1}{2} \int_{\Omega}\left(|\nabla u|^{2}+c(x) u^{2}\right) \mathrm{d} x-\int_{\Omega} F(x, u) \mathrm{d} x-\oint_{\partial \Omega} G(x, u) \mathrm{d} \sigma \\
& \geq \frac{1}{2}\|u\|_{c}^{2}-\frac{1}{2}(\lambda-\varepsilon) \int_{\Omega} u^{2} \mathrm{~d} x-\frac{1}{2}(\mu-\varepsilon) \oint_{\partial \Omega} u^{2} \mathrm{~d} \sigma-\int_{\Omega} f_{0} \mathrm{~d} x-\oint_{\partial \Omega} g_{0} \mathrm{~d} \sigma
\end{aligned}
$$

Assume that $\|u\|_{c} \rightarrow \infty$, then using the continuity of the trace operator from $H^{1}(\Omega)$ into $L^{2}(\partial \Omega)$, we obtain either $\|u\|_{\partial} \rightarrow \infty$, or $\|u\|_{\partial} \leq M_{2}$, where $M_{2}$ is a positive constant.

Case 1 As $\|u\|_{\partial} \leq M_{2}$, by Equation (3.5), we obtain

$$
\begin{aligned}
\Phi(u) & \geq \frac{1}{2}\|u\|_{c}^{2}-\frac{1}{2}\left(\frac{\lambda}{\lambda_{1}}-\frac{\varepsilon}{\lambda_{1}}\right)\|u\|_{c}^{2}-\frac{1}{2}(\mu-\varepsilon) \oint_{\partial \Omega} u^{2} \mathrm{~d} \sigma-\int_{\Omega} f_{0} \mathrm{~d} x-\oint_{\partial \Omega} g_{0} \mathrm{~d} \sigma \\
& \geq\left(1-\frac{\lambda}{\lambda_{1}}+\frac{\varepsilon}{\lambda_{1}}\right)\|u\|_{c}^{2}-\frac{1}{2}(\mu-\varepsilon) \oint_{\partial \Omega} u^{2} d \sigma-\int_{\Omega} f_{0} d x-\oint_{\partial \Omega} g_{0} d \sigma .
\end{aligned}
$$

Hence, we obtain that $\Phi(u)$ is coercive on $H^{1}(\Omega)$ since $\lambda<\lambda_{1}$.

Case2 As $\|u\|_{\partial} \rightarrow \infty$, we have

$$
\begin{aligned}
\Phi(u) & \geq\left(1-\frac{\lambda}{\lambda_{1}}+\frac{\varepsilon}{\lambda_{1}}\right)\|u\|_{c}^{2}-\frac{1}{2}(\mu-\varepsilon) \oint_{\partial \Omega} u^{2} \mathrm{~d} \sigma-\int_{\Omega} f_{0} \mathrm{~d} x-\oint_{\partial \Omega} g_{0} \mathrm{~d} \sigma \\
& \geq\left(1-\frac{\lambda}{\lambda_{1}}-\frac{\mu}{\mu_{1}}+\frac{\varepsilon}{\lambda_{1}}+\frac{\varepsilon}{\mu_{1}}\right)\|u\|_{c}^{2}-\int_{\Omega} f_{0} \mathrm{~d} x-\oint_{\partial \Omega} g_{0} \mathrm{~d} \sigma
\end{aligned}
$$

By H3), we obtain $\mu_{1} \lambda_{1}-\mu_{1} \lambda-\mu \lambda_{1}>0$, then $\Phi(u)$ is coercive on $H^{1}(\Omega)$.

Hence we obtain that the functional $\Phi(u)$ is bounded from below, and every (PS) sequence $\left\{u_{n}\right\}$ is bounded in $H^{1}(\Omega)$. From Lemma 3.1, we obtain that $\Phi(u)$ satisfies (PS) condition and is bounded from below.

The Proof of Theorem 2.2 We write $L^{2}(\Omega)=V \oplus V^{\perp}$, where

$$
V=E\left(\lambda_{1}\right) \oplus E\left(\lambda_{2}\right) \oplus \cdots \oplus E\left(\lambda_{k}\right) \text { and } V^{\perp}=\overline{E\left(\lambda_{k+1}\right) \oplus E\left(\lambda_{k+2}\right) \oplus \cdots} .
$$

Hence, we have

$$
H^{1}(\Omega)=\left(V \cap H^{1}(\Omega)\right) \oplus\left(V^{\perp} \cap H^{1}(\Omega)\right) .
$$


Since $\left(V \cap H^{1}(\Omega)\right)$ is a finite dimensional space, by [2], we obtain that for given $\delta>0$, there is a $\rho_{1}>0$ such that

$$
u \in\left(V \cap H^{1}(\Omega)\right),\|u\|_{c} \leq \rho_{1} \Rightarrow|u(x)| \leq \delta \text {, a.e. } x \in \Omega .
$$

Hence, for each $u \in\left(V \cap H^{1}(\Omega)\right),\|u\|_{c} \leq \rho_{1}$, by H4), we have

$$
\begin{aligned}
\Phi(u) & =\frac{1}{2}\|u\|_{c}^{2}-\int_{\Omega} F(x, u) \mathrm{d} x-\oint_{\partial \Omega} G(x, u) \mathrm{d} \sigma \leq \frac{1}{2}\|u\|_{c}^{2}-\frac{1}{2}(\alpha-\varepsilon) \int_{\Omega} u^{2} \mathrm{~d} x-\frac{1}{2}(a-\varepsilon) \oint_{\partial \Omega} u^{2} \mathrm{~d} \sigma \\
& \leq \frac{1}{2}\left(1-\frac{a}{\mu_{1}}+\frac{\varepsilon}{\mu_{1}}\right)\|u\|_{c}^{2}-\frac{1}{2}(\alpha-\varepsilon) \int_{\Omega} u^{2} \mathrm{~d} x \leq \frac{1}{2}\left(1-\frac{a}{\mu_{1}}-\frac{\alpha}{\lambda_{k}}+\frac{\varepsilon}{\mu_{1}}+\frac{\varepsilon}{\lambda_{k}}\right)\|u\|_{c}^{2}
\end{aligned}
$$

We have for $\varepsilon>0$ sufficiently small,

$$
\frac{1}{2}\left(1-\frac{a}{\mu_{1}}-\frac{\alpha}{\lambda_{k}}+\frac{\varepsilon}{\mu_{1}}+\frac{\varepsilon}{\lambda_{k}}\right)<0 .
$$

Therefore, we obtain that $\Phi(u) \leq 0$.

On the other hand, let $\Omega_{1}=\{x \in \bar{\Omega}:|u(x)| \leq \delta\}$ and $\Omega_{2}=\{x \in \bar{\Omega}:|u(x)|>\delta\}$, then for every $u \in\left(V^{\perp} \cap H^{1}(\Omega)\right)$ with $|u(x)| \leq \delta$, by H4), we obtain that

$$
\int_{\Omega}\left(\frac{1}{2}(\beta+\varepsilon) u^{2}-F(x, u)\right) \mathrm{d} x \geq 0 \text {, and } \oint_{\left(\partial \Omega \cap \Omega_{1}\right)}\left(\frac{1}{2}(b+\varepsilon) u^{2}-G(x, u)\right) \mathrm{d} x \geq 0 .
$$

Combining Equation (3.4), Equation (3.6), H1) and H2), we have

$$
\begin{aligned}
\Phi(u) & =\frac{1}{2}\|u\|_{c}^{2}-\int_{\Omega} F(x, u) \mathrm{d} x-\oint_{\partial \Omega} G(x, u) \mathrm{d} \sigma \\
& =\frac{1}{2}\|u\|_{c}^{2}-\int_{\Omega_{1}} F(x, u) \mathrm{d} x-\oint_{\left(\partial \Omega \cap \Omega_{1}\right)} G(x, u) \mathrm{d} \sigma-\int_{\Omega_{2}} F(x, u) \mathrm{d} x-\oint_{\left(\partial \Omega \cap \Omega_{2}\right)} G(x, u) \mathrm{d} \sigma \\
& \geq \frac{1}{2}\|u\|_{c}^{2}-\frac{(\beta+\varepsilon)}{2} \int_{\Omega} u^{2} \mathrm{~d} x-\frac{(b+\varepsilon)}{2} \oint_{\partial \Omega} u^{2} \mathrm{~d} \sigma-c_{1}\|u\|_{c}^{q_{1}}-c_{2}\|u\|_{\partial}^{q_{2}}-\int_{\Omega} f_{0} \mathrm{~d} x-\oint_{\partial \Omega} g_{0} \mathrm{~d} \sigma \\
& \geq \frac{1}{2}\left(1-\frac{b}{\mu_{1}}-\frac{\varepsilon}{\mu_{1}}\right)\|u\|_{c}^{2}-\frac{1}{2}(\beta+\varepsilon) \int_{\Omega} u^{2} \mathrm{~d} x-c_{1}\|u\|_{c}^{q_{1}}-c_{2}\|u\|_{c}^{q_{2}}-\int_{\Omega} f_{0} \mathrm{~d} x-\oint_{\partial \Omega} g_{0} \mathrm{~d} \sigma \\
& \geq \frac{1}{2}\left(1-\frac{b}{\mu_{1}}-\frac{\beta}{\lambda_{k+1}}-\frac{\varepsilon}{\mu_{1}}-\frac{\varepsilon}{\lambda_{k+1}}\right)\|u\|_{c}^{2}-c_{1}\|u\|_{c}^{q_{1}}-c_{2}\|u\|_{c}^{q_{2}}-\int_{\Omega} f_{0} \mathrm{~d} x-\oint_{\partial \Omega} g_{0} \mathrm{~d} \sigma
\end{aligned}
$$

From Equation (2.6), we have, for $\varepsilon>0$ sufficiently small

$$
\frac{1}{2}\left(1-\frac{b}{\mu_{1}}-\frac{\beta}{\lambda_{k+1}}-\frac{\varepsilon}{\mu_{1}}-\frac{\varepsilon}{\lambda_{k+1}}\right)>0 .
$$

Since $q_{1}, q_{2}>2$, we can choose $\rho_{2}>0$ sufficiently small and $u \in\left(V^{\perp} \cap H^{1}(\Omega)\right)$, $\|u\|_{c} \leq \rho_{2}$, such that the functional $\Phi(u) \geq 0$.

By Lemma 3.2, we obtain that $\Phi(u)$ satisfies (PS) condition and is bounded from below. If $\inf _{u \in H^{1}(\Omega)} \Phi(u)<0$, then by Lemma 2.1, $\Phi$ possesses two nonzero critical point. From (3.3), we obtain that there exist two nontrivial weak solutions for the problem (1.1).

\section{Conclusion}

Using Local Linking Theorem, we obtain the existence of two nontrival weak solutions for the problem (1.1) 
which the nonlinearites $f(x, u)$ and $g(x, u)$ are compared with higher Neumann eigenvalue and the first Steklov eigenvalue.

\section{Acknowledgements}

This paper was supported by Shanghai Natural Science Foundation Project (No. 11ZR1424500) and Shanghai Leading Academic Discipline Project (No. XTKX2012).

\section{REFERENCES}

[1] M. W. Steklov, "Sur les Problemes Fondamentaux de la Physique Mathematique," Annales Scientifiques de l'École Normale Supérieure, Vol. 19, No. 1, 1902, pp. 455-490.

[2] G. Auchmuty, "Steklov Eigenproblems and Representation of Solutions of Elliptic Boundary Value Problems,” Numerical Functional Analysis and Optimization, Vol. 25, No. 3-4, 2005, pp. 321-348. http://dx.doi.org/10.1081/NFA-120039655

[3] H. Amann, "Nonlinear Elliptic Equations with Nonlinear Boundary Conditions," Proceedings of the 2nd Scheveningen Conference on Differential Equations, North-Holland Mathematics Studies, Vol. 21, 1976, pp. 43-63. http://dx.doi.org/10.1016/S0304-0208(08)71154-X

[4] J. Mawhin and K. Schmitt, "Upper and Lower Solutions and Semilinear Second Order Elliptic Equations with Nonlinear Boundary Conditions,” Proceedings of the Royal Society of Edinburgh: Section A, Vol. 97, 1984, pp. 199-207. http://dx.doi.org/10.1017/S030821050003198X

[5] H. Brezis and L. Nirenberg, "Remarks on Finding Critical Points," Communications on Pure and Applied Mathematics, Vol. 44, No. 8-9, 1991, pp. 939-963. http://dx.doi.org/10.1002/cpa.3160440808

[6] C. V. Pao, “Nonlinear Parabolic and Elliptic Equations,” Plenum Press, New York, 1992.

[7] N. Mavinga and M. N. Nkashama, "Steklov-Neumann Eigenproblems and Nonlinear Elliptic Equations with Nonlinear Boundary Conditions,” Journal of Differential Equations, Vol. 248, No. 5, 2010, pp. 1212-1229.

http://dx.doi.org/10.1016/j.jde.2009.10.005 